\title{
EFECTO DEL RIEGO POR GOTEO EN EL CRECIMIENTO INICIAL DE TRES CULTIVARES DE ALGODÓN (GOSSYPIUM HIRSUTUM L.)
}

\author{
EFFECT OF DRIP IRRIGATION ON INITIAL GROWTH OF THREE COTTON \\ (GOSSYPIUM HIRSUTUM L.) CULTIVARS
}

\author{
Jesús Rafael Méndez-Natera ${ }^{1}$; Leonardo Lara ${ }^{1}$; José Alexander Gil-Marín ${ }^{1}$
}

\begin{abstract}
RESUMEN
En Venezuela no se tiene mucha información acerca del riego por goteo en el cultivo del algodón, por tal razón se realizó un ensayo cuyo objetivo fue evaluar el efecto del riego por goteo sobre tres caracteres vegetativos y uno reproductivo a los 38 días después de la siembra en tres cultivares de algodón. Se utilizó un diseño de parcelas divididas; las parcelas principales fueron: cuatro dosis de riego (40,60, 80 y 100\% de la evaporación de un tanque tipo A sobre suelo con cubierta vegetal) y las subparcelas los tres cultivares de algodón. Se usaron tres repeticiones. La frecuencia de riego fue de dos días y la fertilización se realizó por fertirrigación utilizando un Venturi y aplicando $1 \mathrm{~kg} / 20$ litros de agua del fertilizante Solub 18-18-18 con microelementos. Se encontró una respuesta diferencial de los cultivares en función de la dosis de riego en los caracteres evaluados. Para los cultivares Cabuyare y Stroman, incrementos en la dosis de riego produjeron plantas más altas y con mayor número de hojas, mientras que para Deltapine 16 esto se observó hasta la dosis de $80 \%$. Incrementos en la dosis de riego produjeron plantas más precoces a floración y con tallos más gruesos en los cultivares Cabuyare y Stroman, mientras que Deltapine 16 siguió una tendencia similar a excepción de la dosis de $60 \%$ que presentó plantas más tardías y con tallos más delgados. Estos resultados indican que el aumento de las dosis de riego produjo incrementos de los caracteres vegetativos y una mayor precocidad en los tres cultivares.
\end{abstract}

Palabras clave: Riego por goteo, algodón, Gossypium hirsutum, tina de evaporación.

\begin{abstract}
In Venezuela, there is a lack of information about drip irrigation in cotton crop, because of the following; an experiment was carried out in order to evaluate the effect of drip irrigation on three vegetative characters and a reproductive one at 38 days after swing in three cotton cultivars. A split plot design was used, the main plots were four irrigation doses, id est, 40, 60, 80 and $100 \%$ of the evaporation of a type A pan on soil with pasture cover and the sub-plot were three cotton cultivars. Three replications were used. A two-day irrigation frequency was used and the fertilization was applied using fertigation method with a Venturi applying $1 \mathrm{~kg}$ of fertilizer Solub 18-18-18 with micronutrients/20 liters of water. A different response of cultivars according to irrigation doses was found in all traits evaluated. For cultivars Cabuyare and Stroman, increments of irrigation doses produced taller plants with more leaves, while for Deltapine 16 this effect was observed up to 80\% irrigation doses. Also, increments of irrigation doses produced early flowering plants with bigger stem diameter in cultivars Cabuyare and Stroman, cultivar Deltapine follow a similar trend, excepting 60\% irrigation doses where late flowering plants occur with lesser stem diameter. These results indicate that increments of irrigation doses produced incremented vegetative traits and early flowering plants of the three cotton cultivars.
\end{abstract}

Key words: Drip irrigation, cotton, Gossypium hirsutum, evaporation pan.

\section{INTRODUCCIÓN}

La falta de agua durante el crecimiento inicial de la planta de algodón puede ocasionar una disminución del rendimiento de algodón en rama debido a la formación inadecuada de las diferentes partes de la planta, id est, tallos, hojas, ramas, bellotas, etc., traducida en términos de un bajo crecimiento y desarrollo de la planta. Longenecker y Erie (1968) indicaron que es de suma importancia que exista un

1 Escuela de Ingeniería Agronómica, Núcleo Monagas, Universidad de Oriente. Avenida Universidad, Campus Los Guaritos, Venezuela. E-mail: jmendezn@ cantv.net y jalexgil@cantv.net

Fecha de Recepción: 13 Enero 2006

Fecha de Aceptación: 20 Febrero 2006 
buen crecimiento en las etapas iniciales de las plantas de algodón de manera de proveer una estructura suficiente de la planta para una pesada fructificación y cualquier práctica que ayudara a lograr este resultado antes de la primera floración sería beneficiosa y que el desarrollo vegetativo, y por lo tanto el suministro de agua, están directamente relacionados con la formación de ramas fructíferas y con los rendimientos de algodón en rama, permaneciendo las demás cosas iguales, una planta más alta es capaz de producir más bellotas debido al mayor número de sitios disponibles para la producción de frutos o ramas fructíferas. La mayoría de estos sitios de fructificación deben ser creados antes de que abran las primeras flores, así que los riegos postemergencia deberían ser planificados de manera que ocurra el mínimo estrés hídrico en las plantas hasta la floración, sin importar la primera época de floración.

Según Lagière (1969), de la siembra al principio de la floración, las necesidades en agua del cultivo del algodón son relativamente reducidas, pues la vegetación, en este período, sólo transpira débiles cantidades de agua por unidad de superficie. Según las regiones, se estima que la evapotranspiración varía de 2 a $4 \mathrm{~mm}$ /día durante este periodo, o sea de 20 a $40 \mathrm{~m}^{3} / \mathrm{ha} /$ día. A causa del débil enraizamiento del algodonero al principio de la vegetación, se tendrá interés en dar riegos ligeros con cierta frecuencia.

El riego puede incrementar la producción de algodón en el mundo, ya sea permitiendo que el cultivo sea desarrollado en regiones áridas donde de otra forma sería imposible cultivarlo o incrementando los rendimientos con riego suplementario bajo condiciones de lluvia. También tiene el potencial de degradar al ambiente dentro y fuera de la finca; la degradación puede limitar o reducir la producción directamente mediante el daño de los recursos usados en la producción, mientras la amenaza de la degradación puede reducir la producción indirectamente mediante las leyes o los acuerdos internacionales encaminados a su prevención. El riego por goteo hace mucho más fácil mantener el contenido de humedad del suelo dentro de límites más angostos de disponibilidad causado por la salinidad y permite así que el algodón sea cultivado en suelos o con agua que de otra forma sería demasiado salina (Gillham et al., 1995).

En el Oriente del país no se tiene mucha información sobre el efecto del riego en el cultivo del algodón. Méndez-Natera et al. (2001) estudiaron el efecto de tres frecuencias de riego por $\operatorname{surcos}(6,9 \mathrm{y}$
12 días) sobre algunos caracteres de las plantas en cuatro cultivares de algodón (Cabuyare, Deltapine 16, Deltapine 61 y Acala 90-1) en el Valle del Río Guarapiche, Maturín, Monagas. Estos autores concluyeron que las frecuencias de riego no afectaron los caracteres de las plantas en los cuatro cultivares de algodón a excepción de la altura de la primera bellota. Asimismo, los cultivares no mostraron diferencias entre sí para la mayoría de los caracteres, incluyendo el número de bellotas/planta y el rendimiento de algodón en rama/planta, los cuales son componentes importantes del rendimiento de algodón en rama/ha.

Debido a la importancia que tiene el crecimiento y desarrollo inicial de las plantas de algodón en los rendimientos finales de algodón en rama, especialmente la altura de la planta y la época de floración el objetivo del siguiente trabajo fue evaluar el efecto del riego por goteo sobre tres caracteres vegetativos y uno reproductivo a los 38 días después de la siembra en tres cultivares de algodón.

\section{MATERIALES Y MÉTODOS}

Este trabajo se realizó en el Campus Los Guaritos, situado geográficamente entre los $9^{\circ} 46^{\prime}$ $46^{\prime \prime}$ latitud norte y $63^{\circ} 11^{\prime} 51^{\prime \prime}$ longitud oeste, a una altura de 63,5 ms.n.m. de la Universidad de Oriente. Se usaron tres cultivares de algodón: Deltapine 16, Cabuyare y Stroman y cuatro dosis de riego: $40 \%$, $60 \%, 80 \%$ y $100 \%$ de la lámina evaporada que se midieron en un tanque evaporímetro tipo $\mathrm{A}$.

La fórmula empleada para el cálculo de la dosis de riego fue:

$$
\begin{aligned}
& \sum E T P=\sum \text { Evaporación de la tina }\left(1^{\mathrm{er}} \mathrm{y} 2^{\mathrm{do}} \text { día }\right) \\
& \begin{array}{l}
d=E T P^{*} 0,80 \\
T=\frac{d^{*} A}{Q}
\end{array}
\end{aligned}
$$

Donde:

ETP: Evapotranspiración calculada mediante la tina tipo A.

d: Lámina de riego.

T: $\quad$ Tratamientos $(100,80,60$ y $40 \%$ de la tina de evaporación).

A: Área que ocupa la planta por cada gotero.

Q: Caudal. 
La evapotranspiración potencial máxima del cultivo (Eo) se obtuvo aplicando la fórmula:

$$
\text { 'Eo }=\mathrm{Ev} \times 0,80 \times \mathrm{Kc}
$$

Donde:

Eo: Evapotranspiración potencial máxima del cultivo en mm/día.

Ev: Evaporación producida por una tina tipo A, medida en mm/día.

0,80: Constante de ajuste al método del tanque tipo A.

Kc: coeficiente del cultivo (varía con la edad del mismo).

El coeficiente del cultivo $(\mathrm{Kc})$ del algodón para diferentes etapas del crecimiento es: para la etapa inicial 0,4-0,5 (20 a 30 días), la etapa de desarrollo 0,7-0,8 (40 a 50 días), la etapa de mitad de estación 1,05-1,25 (50 a 60 días), la última etapa del ciclo 0,8-0,9 (40 a 55 días) y la cosecha 0,65-0,70 (FAO, 2002).

Se empleó un diseño experimental de parcelas divididas con tres repeticiones, siendo las parcelas principales los tratamientos de riego y las subparcelas los cultivares de algodón, para un total de 12 tratamientos. Las parcelas principales estuvieron constituidas por un múltiple de cuatro laterales de $10 \mathrm{~m}$ de longitud, separados a 1,00 m entre lateral, y entre cada múltiple se dejó una distancia de 1,00 m para facilitar el libre tránsito dentro del ensayo. Las subparcelas estuvieron constituidas por cuatro laterales con 6 goteros cada uno, separados por 0,50 m entre sí, considerándose para efectos de evaluación sólo las hileras centrales, mientras que las laterales se consideraron como borduras. Se realizó el análisis de varianza convencional y a las diferencias entre la interacción dosis de riego x cultivar se les realizó un análisis de regresión aplicando los modelos lineal, cuadrático y cúbico (Gómez y Gómez, 1984). El nivel de probabilidad fue de 0,10. Los datos del porcentaje de plantas en floración se transformaron mediante la fórmula $\mathrm{Log}$ (x), donde $\mathrm{x}$ es el dato original. El área total del experimento fue de $528 \mathrm{~m}^{2}$, con un área efectiva de $432 \mathrm{~m}^{2}$, teniendo las parcelas principales un área de $40 \mathrm{~m}^{2}$ y las subparcelas un área de $12 \mathrm{~m}^{2}$.

La siembra se realizó en forma manual, en hileras dobles a una distancia de $0,25 \mathrm{~m}$ entre hileras, 0,75 m entre hileras dobles y $0,50 \mathrm{~m}$ entre plantas dentro de las hileras dobles, para una densidad de 80.000 plantas/ha, las cuales dentro del ensayo dieron un aproximado 3.456 plantas que se traduce en 8 plantas $/ \mathrm{m}^{2}$. Se colocaron 4 semillas por hoyo a una profundidad aproximada de $2 \mathrm{~cm}$. Luego se realizó un raleo de tal manera que quedaron 2 plantas por punto de siembra, es decir, 4 plantas a ambos lados del punto de emisión.

En el control de malezas se aplicó un herbicida postemergente después de la siembra (Dual a razón de 2 1/ha). Además se efectuaron dos limpiezas manuales, la primera a los 30 días y la segunda 60 días después de la siembra. En cuanto al control de plagas, por ataque de bachacos (Atta sexdens), se procedió a aplicar Lebaycid en cada cueva encontrada.

El riego se realizó a presión, utilizando un sistema por goteo constituido principalmente por:

- Bomba: La cual constituye la fuente que suministra energía al sistema.

- Línea de Aducción: Conduce el agua desde la bomba a toda la red de distribución.

- Cabezal de control: Es el conjunto de dispositivos que dominan toda la superficie de la instalación; donde se encuentra el filtro.

- Llave: Controla el paso del flujo. La inyección de fertilizante se realizó utilizando un Venturi que controla el paso de la solución de fertilizante.

- Línea principal: Transporta el agua desde el cabezal de control hasta las unidades de riego. El diámetro será de $25 \mathrm{~mm}$.

- Línea secundaria: Son las que dentro de una unidad de riego sirven a las distintas subunidades. El diámetro será de $25 \mathrm{~mm}$.

- Múltiple: Conjunto de llave, tubos y conexiones que llevan el agua desde la línea secundaria y la distribuye a las diferentes líneas laterales. El diámetro será de media pulgada (1/2").

- Línea lateral: Tubería de polietileno en la cual se encuentran los goteros incorporados del tipo embutido. El diámetro será de $16 \mathrm{~mm}$.

El agua se extrajo de un pozo perforado y fue depositada en un tanque de $40 \mathrm{~m}^{3}$ de capacidad para posteriormente ser succionada por medio de una bomba centrífuga marca FANAB de $1 / 3$ de HP que funciona a 3.480 RPM con una presión media de 25 a 30 PSI con un gasto de 1.000 a 2.000 1/h.

La fertilización se realizó mediante la utilización de un Venturi, el cual transportaba el fertilizante disuelto en 20 litros de agua aproximadamente, a través del sistema de riego. Para esta práctica se utilizaron tres fertilizantes solubles: Urea, Fertisol y $\mathrm{KCl}$, utilizándose una fórmula 
de 12-8-12. Este proceso se llevó a cabo tomando en cuenta el tratamiento menor $(40 \%$ de la lámina evaporada), la cual mediante cálculos se traducía en el menor tiempo, tomándose en cuenta el tiempo que el Venturi tardaba en extraer todo el fertilizante para ser trasportado. Inicialmente se abrían las válvulas y se dejaban llenar las mangueras durante 5 minutos para que la aplicación fuera lo más homogénea posible.

Se determinaron los siguientes caracteres a los 38 días después de la siembra: altura de la planta, número de hojas por planta, diámetro del tallo y porcentaje de plantas en floración.

\section{RESULTADOS Y DISCUSIÓN}

Todos los caracteres presentaron una interacción significativa dosis de riego $\mathrm{x}$ cultivares (Cuadro 1). El cultivar Deltapine 16 presentó respuestas cuadrática, lineal, cúbica y lineal para la altura de la planta, diámetro del tallo, número de hojas por planta y porcentaje de plantas en floración, respectivamente, en función de las dosis de riego. El cultivar Cabuyare presentó una respuesta lineal en todos los caracteres evaluados a excepción del diámetro del tallo donde la respuesta fue cuadrática, mientras el cultivar Stroman presentó una respuesta cuadrática en todos los caracteres excepto para el porcentaje de plantas en floración donde la respuesta fue cúbica. Estos resultados indican las diferencias varietales de los cultivares ante las diferentes dosis de riego. Por otra parte, el porcentaje de plantas en floración presentó los coeficientes de variación más altos, mientras que la altura de la planta y el diámetro del tallo los más bajos.

Incrementos en las dosis de riego ocasionaron incrementos de la altura de la planta en los tres cultivares evaluados, siendo el efecto más marcado en el cultivar Stroman en la dosis de $100 \%$ y el menos marcado para el cultivar Deltapine 16. El cultivar Cabuyare tuvo una respuesta directamente proporcional a las dosis de riego. La dosis de $100 \%$ produjo las plantas más altas, mientras que la dosis de $40 \%$ las plantas más bajas en todos los cultivares (Figura 1).

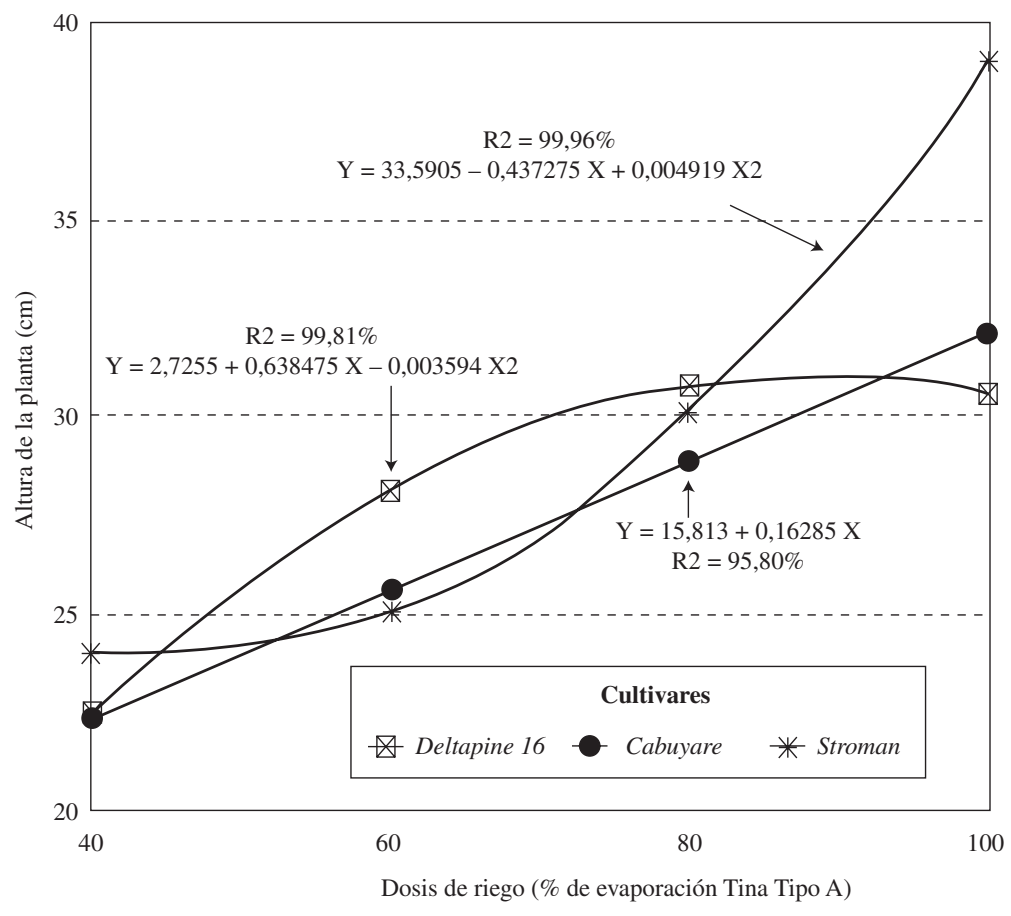

Figura 1. Altura de la planta $(\mathrm{cm})$ de tres cultivares de algodón (Gossypium hirsutum L.) bajo cuatro dosis de riego en el Campus Los Guaritos de la Universidad de Oriente, Maturín, edo. Monagas. Efecto de la interacción dosis de riego x cultivar. 
Cuadro 1

Análisis de varianza y de regresión de la altura de la planta (cm) (AP), diámetro del tallo (mm) (DT), número de hojas por planta (HP) y porcentaje de plantas en floración (PF) a los 38 días después de la siembra de tres cultivares de algodón (Gossypium hirsutum L.) bajo cuatro dosis de riego en el Campus Los Guaritos de la Universidad de Oriente, Maturín, edo. Monagas.

\begin{tabular}{|c|c|c|c|c|c|c|c|c|c|}
\hline \multirow{2}{*}{$\begin{array}{l}\text { Fuente de } \\
\text { Variación }\end{array}$} & \multirow{2}{*}{$\begin{array}{l}\text { Grados de } \\
\text { Libertad }\end{array}$} & \multicolumn{8}{|c|}{ Cuadrados Medios } \\
\hline & & \multicolumn{2}{|l|}{$\mathbf{A P}$} & \multicolumn{2}{|l|}{ DT } & \multicolumn{2}{|l|}{ HP } & \multicolumn{2}{|l|}{$\mathbf{P F} \dagger$} \\
\hline Repetición & 2 & 299,4505 & $*$ & 12,7820 & $*$ & 82,7725 & $*$ & 0,0125 & ns \\
\hline Dosis Riego (DR) & 3 & 201,6720 & $* *$ & 3,7857 & ns & 42,1883 & $* *$ & 0,3797 & ns \\
\hline Error Experim. (a) & 6 & 56,2932 & & 2,0887 & & 11,8897 & & 0,2398 & \\
\hline Cultivares (C) & 2 & 16,8350 & $* *$ & 0,0785 & ns & 0,4040 & ns & 0,0080 & ns \\
\hline $\mathrm{DR} * \mathrm{C}$ & 6 & 20,2935 & $*$ & 0,3058 & $* *$ & 3,0340 & $*$ & 0,1012 & $* *$ \\
\hline Deltapine 16 en DR & 3 & 44,9907 & $*$ & 1,0186 & $*$ & 10,1728 & $*$ & 0,0819 & ns \\
\hline Reg Lineal & 1 & 109,9177 & $*$ & 2,8253 & $*$ & 22,1798 & $*$ & 0,1878 & $* *$ \\
\hline Reg Cuadrática & 1 & 24,7969 & $* *$ & 0,1728 & ns & 3,4992 & $*$ & 0,0480 & ns \\
\hline Reg Cúbica & 1 & 0,2574 & ns & 0,0577 & ns & 4,8394 & $*$ & 0,0099 & ns \\
\hline Cabuyare en DR & 3 & 55,3683 & $*$ & 1,5971 & $*$ & 18,6144 & $*$ & 0,3375 & $*$ \\
\hline Reg Lineal & 1 & 159,1207 & $*$ & 4,1976 & $*$ & 51,2266 & $*$ & 1,0039 & $*$ \\
\hline Reg Cuadrática & 1 & 6,8857 & ns & 0,4219 & $* *$ & 1,3872 & ns & 0,0001 & $\mathrm{~ns}$ \\
\hline Reg Cúbica & 1 & 0,0984 & ns & 0,1717 & ns & 3,2294 & ns & 0,0086 & ns \\
\hline Stroman en DR & 3 & 141,9001 & $*$ & 1,7817 & $*$ & 19,4691 & $*$ & 0,1626 & $*$ \\
\hline Reg Lineal & 1 & 379,0609 & $*$ & 4,0093 & $*$ & 48,3304 & $*$ & 0,1272 & ns \\
\hline Reg Cuadrática & 1 & 46,4527 & $*$ & 1,1347 & $*$ & 8,9269 & $*$ & 0,1793 & $* *$ \\
\hline Reg Cúbica & 1 & 0,1865 & ns & 0,2010 & ns & 1,1502 & ns & 0,1811 & \\
\hline Error Experim. (b) & 16 & 6,0854 & & 0,1367 & $*$ & 1,0624 & & 0,0459 & \\
\hline Total & 35 & & & & & & & & \\
\hline $\begin{array}{l}\dagger: \text { Datos transformado } \\
*: \text { Significativo }(\mathrm{p} \leq 0 \\
* *: \text { Significativo }(\mathrm{p} \leq\end{array}$ & ante $\log (\mathrm{x})$ & & & & & & & & \\
\hline \multicolumn{10}{|c|}{ ns : No Significativo $(\mathrm{p}>0,10)$} \\
\hline C.V. (a) $(\%)$ & & 26,55 & & 26,93 & & 35,78 & & 36,72 & \\
\hline C.V. (b) (\%) & & 8,73 & & 6,89 & & 10,70 & & 16,05 & \\
\hline
\end{tabular}

La dosis de $100 \%$ produjo las plantas con tallos más gruesos, mientras que la dosis de $40 \%$ produjo plantas con un grosor menor de los tallos. Stroman tuvo los tallos más gruesos que el resto de los cultivares en las dosis de 40 y $100 \%$, mientras que tuvo los tallos menos gruesos en las dosis de 60 y $80 \%$ (Figura 2). La dosis de $100 \%$ produjo plantas con más hojas en los cultivares Cabuyare y Stroman, mientras que la de $80 \%$ produjo plantas con más hojas en el cultivar Deltapine 16. La menor cantidad de hojas por planta en los tres cultivares ocurrió en la dosis de 40\% (Figura 3).
La Figura 4 muestra la interacción dosis de riego x cultivar para el porcentaje de plantas en floración a los 38 días después de la siembra. En general, las plantas de algodón en los tres cultivares fueron más precoces en floración con la mayor cantidad de agua aplicada (dosis de 100\%), seguido por la dosis de $80 \%$. La disminución de la cantidad de agua aplicada al cultivo (dosis de $40 \%$ ) produjo plantas más tardías en los cultivares Cabuyare y Deltapine 16, mientras que esto sucedió para el cultivar Stroman en la dosis de $60 \%$. Las dosis de 40 y $60 \%$ permitieron discriminar mejor entre 


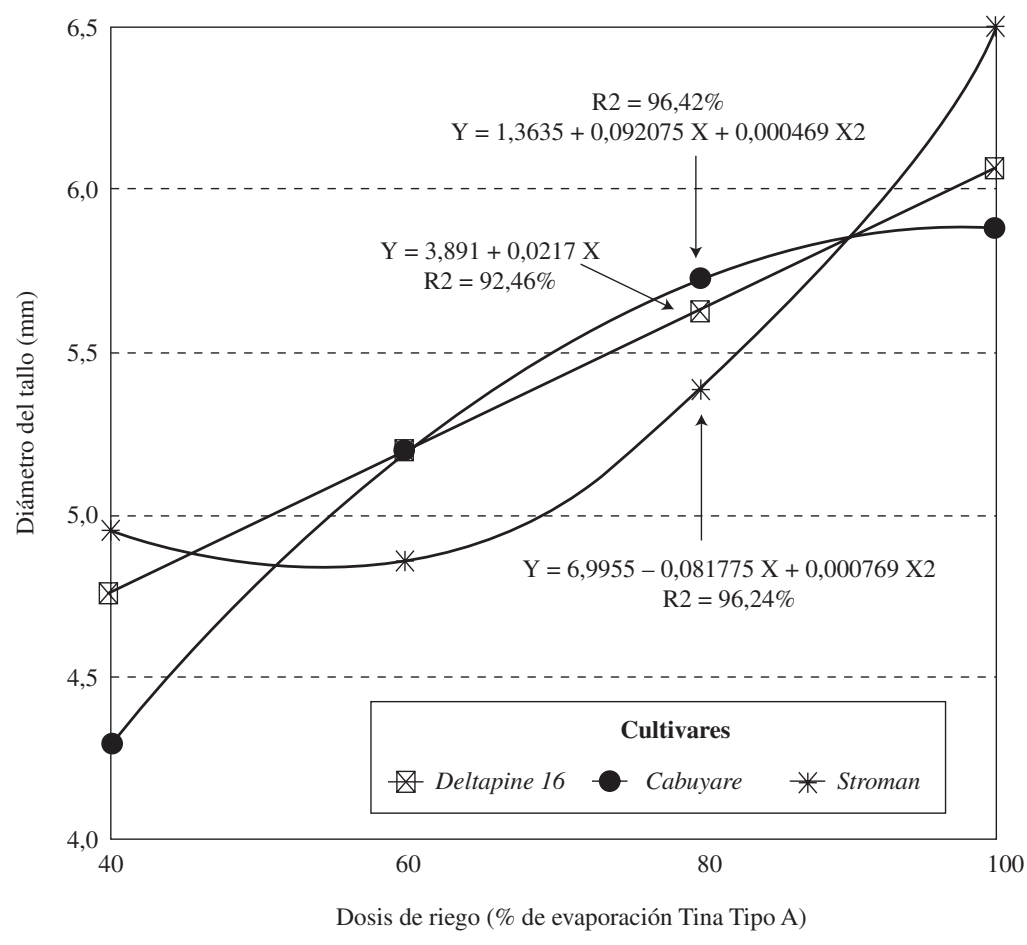

Figura 2. Diámetro del tallo (mm) de tres cultivares de algodón (Gossypium hirsutum L.) bajo cuatro dosis de riego en el Campus Los Guaritos de la Universidad de Oriente, Maturín, edo. Monagas. Efecto de la interacción dosis de riego x cultivar.

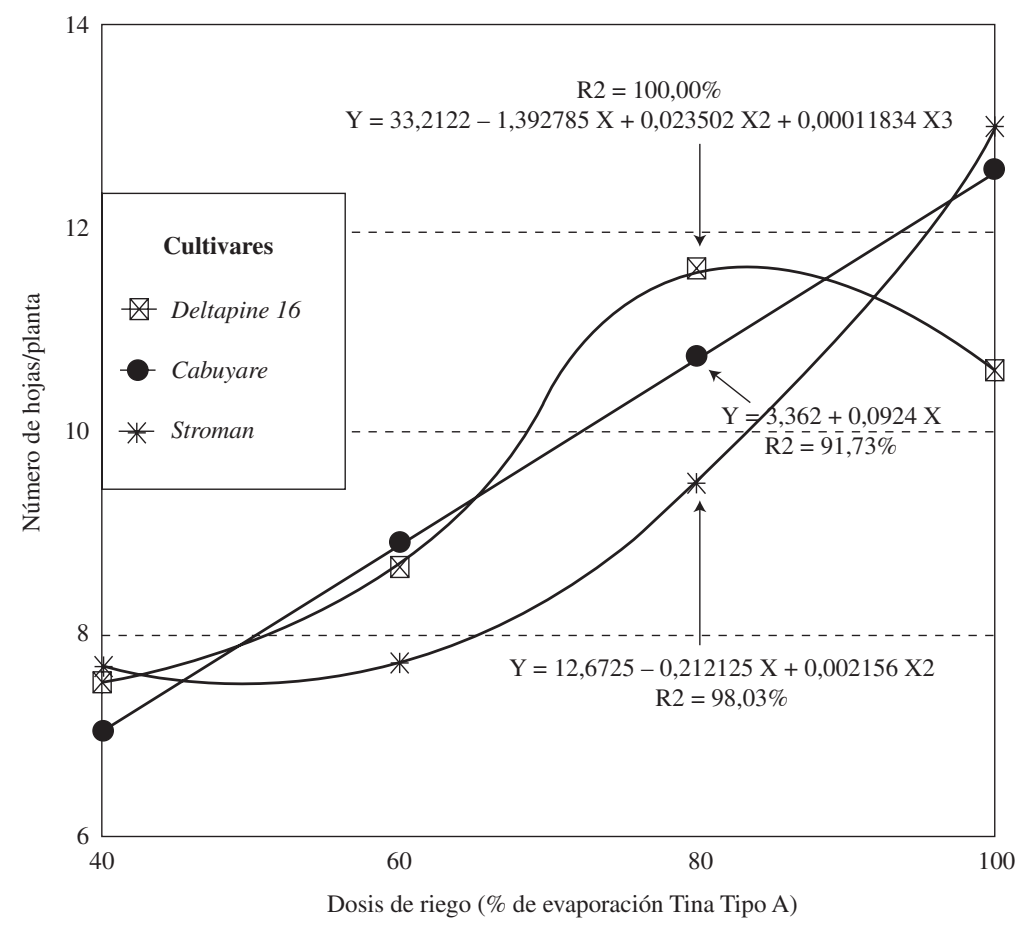

Figura 3. Número de hojas por planta de tres cultivares de algodón (Gossypium hirsutum L.) bajo cuatro dosis de riego en el Campus Los Guaritos de la Universidad de Oriente, Maturín, edo. Monagas. Efecto de la interacción dosis de riego x cultivar. 
cultivares para la precocidad, es decir, en estas dos dosis se apreciaron las mayores diferencias varietales en cuanto al número de plantas en floración a los 38 días después de la siembra. Estos resultados sugieren que la aplicación de una dosis (lámina) de agua correspondiente al 40 o 60\% de la evaporación de la tina tipo A podría ser utilizada para cribar y seleccionar genotipos de algodón con una estabilidad o tolerancia a la sequía en relación al ciclo del cultivo al menos en las primeras etapas del mismo, es decir, desde la siembra hasta el inicio de floración.

En general, las ecuaciones de regresión lineal, cuadrática y cúbica ajustaron bastante bien la relación existente entre las dosis de riego y los caracteres evaluados en los tres cultivares. De las doce ecuaciones estimadas, diez de ellas tuvieron coeficientes de determinación superiores al $90 \%$, inclusive en una de ellas, el valor fue de $100 \%$. Las otras dos ecuaciones tuvieron coeficientes de determinación de $73,91 \%$ (Stroman) y 76,43\% (Deltapine 16) para el porcentaje de plantas en floración a los 38 días después de la siembra.

Se observó que incrementos en las dosis de riego produjeron plantas más altas, con tallos más gruesos y más hojas, así como una mayor cantidad de plantas en floración a los 38 días después de la siembra para los tres cultivares, es decir, entre mayor la dosis de riego, mayor fue la precocidad de las plantas, indicando que en las dosis menores (40 y $60 \% \%$ ) hubo un retardo en el crecimiento manifestado por plantas más pequeñas, con tallos delgados y menor cantidad de hojas y retardo en la floración. Alves et al. (2005) encontraron similares resultados pero en un estudio realizado para evaluar el efecto de niveles de riego sobre el crecimiento de árboles jóvenes de lima Tahití bajo condiciones de campo con tratamientos de riego por goteo basados en $100 \%$ y $50 \%$ de la evapotranspiración del cultivo y cultivo no regado, estos autores encontraron que las plantas regadas con $100 \%$ mostraron una mayor área foliar, un mayor diámetro del tallo y una mayor altura de plantas y que los valores intermedios de estas variables se observaron en el tratamiento de $50 \%$ comparado con $100 \%$ y sin riego e indicaron que el desarrollo de las plantas sin riego fue probablemente disminuido debido a los bajos valores de la conductancia estomática y la asimilación de $\mathrm{CO}_{2}$ y concluyeron que la reposición del $100 \%$ del agua evapotranspirada resultó en un mejor desenvolvimiento vegetativo y reproductivo de las plantas jóvenes de lima Tahití

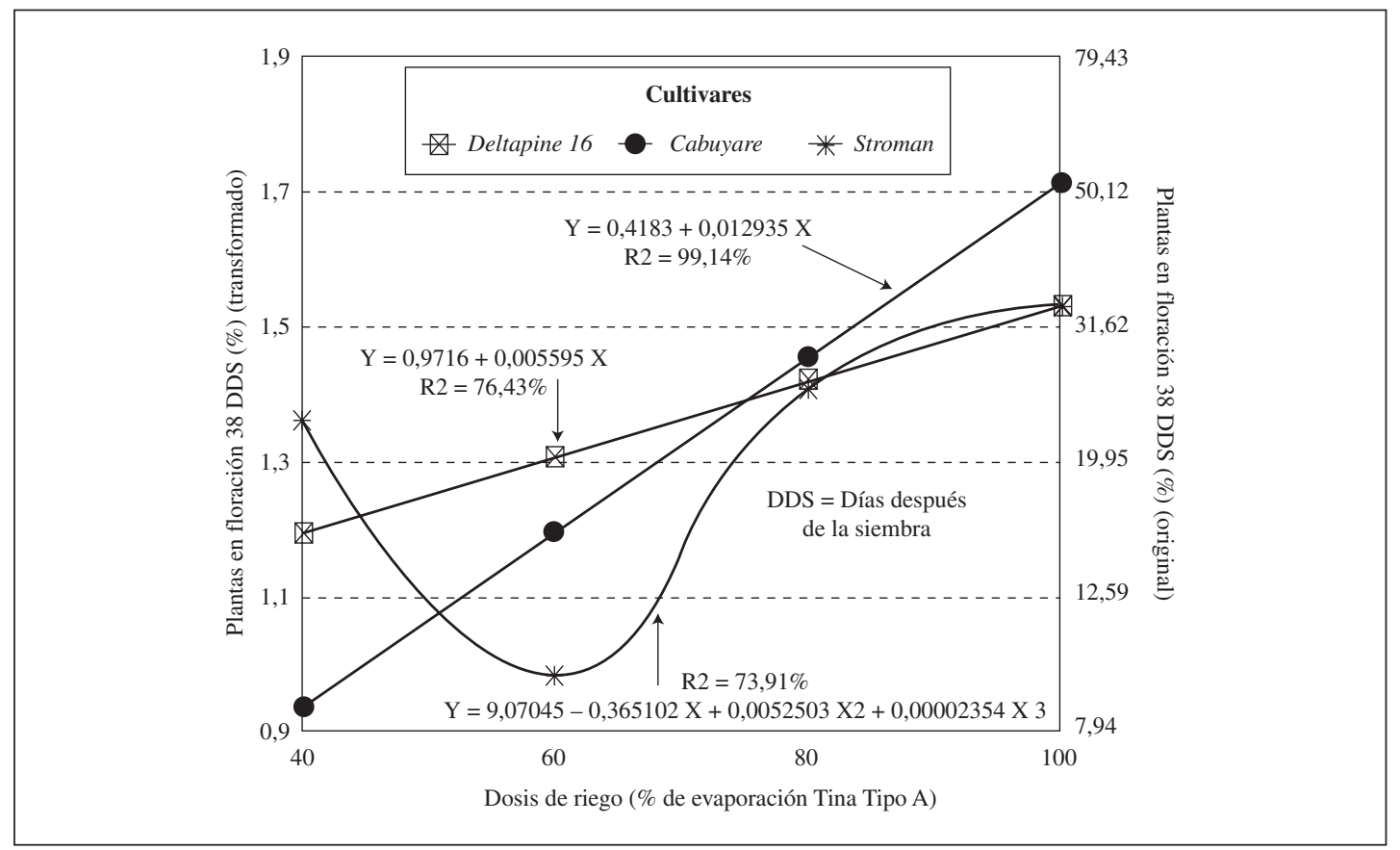

Figura 4. Porcentaje de plantas en floración a los 38 días después de la siembra de tres cultivares de algodón (Gossypium hirsutum L.) bajo cuatro dosis de riego en el Campus Los Guaritos de la Universidad de Oriente, Maturín, edo. Monagas. Efecto de la interacción dosis de riego x cultivar. 
en condiciones de campo, mientras que Santana et al. (2004) realizaron un experimento para evaluar el desarrollo de dos cultivares de café usando cinco niveles de riego (20, 40 y $60 \mathrm{kPa}$ establecido usando tensiómetros; sistema Irrigas con una tensión del agua del suelo definido por su sensor y cultivo sin riego) y encontraron que en los primeros 316 días después del trasplante del café no hubo diferencias en el comportamiento de los dos cultivares regados en tensiones de agua del suelo de 20, 40 y $60 \mathrm{kPa}$ y con el sistema Irrigas, pero estos sí fueron superiores al tratamiento sin riego para los caracteres índice de área foliar, número de hojas, diámetro del tallo y altura de planta, aunque Scalco et al. (2002), estudiando tensiones de 20,80, 140 y $200 \mathrm{kPa}$, concluyeron que una tensión de $20 \mathrm{kPa}$ presentó los mayores valores de crecimiento del cultivar de café Rubí a los 300 días después del trasplante, resultado similar al obtenido en este ensayo.

En este trabajo, la aplicación de riego por goteo a $100 \%$ de la evaporación incrementó la altura de la planta en los tres cultivares de algodón, lo cual podría garantizar la obtención de altos rendimientos de algodón en rama.

En otras palabras, las menores dosis de riego causaron una reducción del crecimiento y desarrollo de las plantas de algodón. Al respecto, FAO (2002) indicó que en la siembra del cultivo de algodón debería existir un contenido de humedad del suelo adecuado para la germinación y el establecimiento y durante el periodo vegetativo el contenido de humedad del suelo sobre la profundidad de las raíces de aproximadamente $0,75 \mathrm{~m}$ no debería estar por debajo del $50 \%$ de agotamiento. Un agotamiento mayor del agua disponible del suelo (hasta 75\%) restringirá el crecimiento vegetativo. Estos resultados indican que las dosis de 40 y $60 \%$ fueron ineficientes en promover el crecimiento y desarrollo de las plantas. Esto pudo deberse, entre otras razones, a que la restauración de sólo el 40 y $60 \%$ de la evaporación ocurrida no es suficiente para sostener el crecimiento armónico de la planta de algodón. Adicionalmente, estas evaluaciones se realizaron a los 38 días después de la siembra, es decir, riegos en la etapa temprana del cultivo. Al respecto, Dalton et al. (2001) indicaron que existe una diferencia obvia entre la eficiencia de la aplicación de los riegos en la estación temprana de crecimiento y los riegos aplicados posteriores a esta etapa, porque, ciertamente, los riegos presiembra o los primeros riegos al inicio del cultivo son significativamente menos eficientes, lo cual se debe más probablemente a la naturaleza más permeable del suelo en esta etapa.

De lo expuesto por Lagière, se puede inferir que en este experimento donde se regaba cada dos días de acuerdo a la evaporación observada, el tratamiento de $40 \%$ no suministró el agua necesaria para el crecimiento de las plantas sin importar que las necesidades de agua del cultivo fueran menores. Como se mencionó al comienzo, los mejores resultados se encontraron con la dosis más alta de riego (100\%). Resultados similares pero en relación al rendimiento de algodón en rama y uso eficiente del agua fueron reportados por O'Brien y Hulme (2002), quienes para tratar de determinar una forma confiable para programar los riegos para un sistema de riego por goteo en el cultivo de algodón con alta precisión realizaron un experimento durante dos estaciones en Waren, Australia. Calculando el uso de agua por el cultivo de algodón usando los datos de evapotranspiración diaria de una estación climática y un factor del cultivo, el cultivo se regó a cuatro diferentes niveles y cada tratamiento fue regado cada día con una porción del uso estimado del agua por el cultivo del día anterior de acuerdo a los siguientes tratamientos: $125,100,75$ y $50 \%$ del uso del agua por el cultivo. Estos autores encontraron que en ambas estaciones el cultivo de algodón en el tratamiento de $100 \%$ tuvo el rendimiento más alto (balas de algodón/ha), dio el mejor retorno económico e hizo un uso más eficiente del agua aplicada y concluyeron que si el agua es escasa, entonces es mejor errar por defecto (subirrigación), mientras que si el agua es abundante entonces es mejor errar por exceso (sobreirrigación), pero fue importante observar que hubo una penalidad en el rendimiento si $25 \%$ más de agua de la requerida por el cultivo fue aplicada.

\section{CONCLUSIONES}

Para los cultivares Cabuyare y Stroman, incrementos en la dosis de riego produjeron plantas más altas y con mayor número de hojas, mientras que para Deltapine 16 esto se observó hasta la dosis de $80 \%$. Incrementos en la dosis de riego produjeron plantas más precoces a floración y con tallos más gruesos en los cultivares Cabuyare y Stroman, mientras que Deltapine 16 siguió una tendencia similar a excepción de la dosis de $60 \%$ 
que presentó plantas más tardías y con tallos más delgados.

Estos resultados indican que el aumento de las dosis de riego produjo incrementos de los caracteres vegetativos y una mayor precocidad en los tres cultivares y que el cultivo debería regarse tomando en cuenta la restauración del 80 al 100\%

\section{LITERATURA CITADA}

ALVES, J.; DA SILVA, C. R.; RIBEIRO, R. V.; DA SILVA, T. J. A.; FOLEGATTI, M. V. 2005. Crescimento de plantas jovens de limeira ácida 'Tahiti' sob lâminas de irrigação. Engenharia Agrícola, Jaboticabal 25 (1): 170-178.

DALTON, P.; RAINE, S.; BROADFOOT, K. 2001. Best management practices for maximizing whole farm irrigation efficiency in the cotton industry. Final Report for CRDC Project NEC2C. National Centre for Engineering in Agriculture Publication 179707/2, USQ, Toowoomba. $81 \mathrm{p}$.

FOOD AND AGRICULTURE ORGANIZATION (FAO). 2002. Crop Water Management: Cotton. http://www.fao. org/ag/agl/aglw/cropwater/cotton.stm. Última visita: 05 de enero de 2006.

GILLHAM, F. E. M.; BELL, T. M.; ARIN, T.; MATTHEWS, G. A.; LE RUMEUR, C. AND BRIAN HEAR, A. 1995. Cotton production prospects for the next decade. World Bank Technical Paper Number 287. The World Bank, Washington D.C. U. S. A. 277 pp.

GÓMEZ, K. A.; GÓMEZ, A. A. 1984. Statistical procedures for agricultural research. John Wiley \& Sons. New York. U. S. A. 680 p.

LAGIÈRE, R. 1969. El algodón. Traducido por V. Ripoll. Editorial Blume, Barcelona, España. 292 pp. de la evaporación determinada por la tina de evaporación tipo A.

\section{AGRADECIMIENTO}

Al Consejo de Investigación de la Universidad de Oriente por el soporte dado al primer autor.

LONGENECKER, D. E.; ERIE, L. J. 1968. Irrigation water management. In Advances in Production and Utilization of Quality. Cotton: Principles and Practices. The Iowa State University Press. Iowa. U. S. A. pp. 321-345.

MÉNDEZ-NATERA, J. R.; SALAZAR-BRITO, R. S.; MERAZO-PINTO, J. F.; GIL-MARÍN, J. A. Y KHANPRADO, L. 2001. Efecto de tres frecuencias de riego sobre algunos caracteres de la planta en cuatro cultivares de algodón (Gossypium hirsutum L.) tipo Upland. Revista Científica UDO Agrícola 1 (1): 48-55.

O'BRIEN. B.; HULME, P. 2002. Irrigation scheduling for cotton under drip. The Australian Cottongrower 23 (1): 72-76.

SANTANA, M. S.; DA S. OLIVEIRA, C. A.; QUADROS, M. 2004. Crescimento inicial de duas cultivares de cafeeiro adensado influenciado por níveis de irrigação localizada. Engenharia Agrícola, Jaboticabal 24 (3): 644-653.

SCALCO, M. S.; MORAIS, A. R.; COLOMBO, A.; CARVALHO, C. H. M.; FARIA, M.A.; MELO, L.Q.; DA SILVA, E. 2002. Influência de diferentes critérios de irrigação e densidades de plantio sobre o crescimento inicial do cafeeiro. In: Simpósio Brasileiro de Pesquisa em Cafeicultura Irrigada, Araguari. Anais. Uberlândia. Universidade Federal de Uberlândia. p. 150-55. 